\title{
Cardiac actin changes in the actomyosin interface have different effects on myosin duty ratio
}

\begin{tabular}{|r|l|}
\hline Journal: & Biochemistry and Cell Biology \\
\hline Manuscript ID & bcb-2017-0136.R1 \\
\hline Danuscript Type: & Article \\
\hline Complete List of Authors: & $\begin{array}{l}\text { Liu, Haidun; University of Guelph College of Biological Science, Molecular } \\
\text { and Cellular Biology; Univeristy of Guelph, Centre for Cardiovascular } \\
\text { Investigations } \\
\text { Henein, Mary; University of Guelph College of Biological Science, Molecular } \\
\text { and Cellular Biology; Univeristy of Guelph, Centre for Cardiovascular } \\
\text { Investigations } \\
\text { Anillo, Maria; University of Guelph College of Biological Science, Molecular } \\
\text { and Cellular Biology; Univeristy of Guelph, Centre for Cardiovascular } \\
\text { Investigations } \\
\text { Dawson, John F.; University of Guelph College of Biological Science, } \\
\text { Molecular and Cellular Biology; University of Guelph, Centre for } \\
\text { Cardiovascular Investigations }\end{array}$ \\
\hline Is the invited manuscript for & Nonsideration in a Special \\
Issue? : & N/A \\
\hline Keyword: & $\begin{array}{l}\text { hypertrophic cardiomyopathy, cardiac actin, mutations, actomyosin, duty } \\
\text { ratio }\end{array}$ \\
\hline
\end{tabular}




\section{Cardiac actin changes in the actomyosin interface have different}

\section{effects on myosin duty ratio}

6 Haidun Liu, Mary Henein, Maria Anillo, and John F. Dawson

7 Contact: jdawso01@uoguelph.ca

8 Department of Molecular and Cellular Biology and the Centre for Cardiovascular Investigations, University of

9 Guelph, Guelph, Ontario, N1G 2W1, Canada. 


\section{Abstract}

12 Hypertrophic cardiomyopathy (HCM) is an inherited cardiovascular disease (CD) that commonly

13 causes an increased size of cardiomyocytes in the left ventricle. The proteins myosin and actin

14 interact in the myocardium to produce contraction through the actomyosin ATPase cycle. The

15 duty ratio $(r)$ of myosin is the proportion of the actomyosin ATPase cycle that myosin is bound

16 to actin and does work. A common hypothesis is that HCM mutations increase contraction in

17 cardiac sarcomeres; however, the available data is not clear on this connection. Based on

18 previous work with human $\alpha$-cardiac actin (ACTC), we hypothesize that HCM-linked ACTC

19 variants with alterations near the myosin binding site have an increased $r$, producing more force.

20 Myosin duty ratios using human ACTC variant proteins were calculated with myosin ATPase

21 activity and in vitro motility data. We found no consistent changes in the duty ratio of the ACTC

22 variants, suggesting that other factors are involved in the development of HCM when ACTC

23 variants are present.

25 Keywords: hypertrophic cardiomyopathy, cardiac actin, mutations, actomyosin, duty ratio 


\section{Introduction}

Cardiovascular disease (CD) is the most costly global disorder and has become the

29 greatest cause of mortality in the world, being responsible for one third of deaths worldwide with

30 immense economic costs ("WHO | Cardiovascular diseases (CVDs)" 2016). In Canada alone,

31 where there are more than 35 million people, over $\$ 20$ billion is spent annually to treat CD

32 (Tarride et al. 2009). Among the many forms of CD, hypertrophic cardiomyopathy (HCM) is an

33 inherited disease that, among a heterogeneous spectrum of abnormalities (Pandian et al. 2015),

34 commonly results in the overgrowth of the left ventricle and is a leading cause of sudden cardiac

35 death in individuals under the age of 30 (Seidman and Seidman 2001, Gajewski and Saul 2010,

36 Maron et al. 2014).

37 The functional units of the heart muscle are sarcomeres which contain actin and myosin

38 proteins. These interact together in a power stroke, shortening the sarcomeres, leading to muscle

39 contraction. The power stroke requires the hydrolysis of ATP by myosin when bound to actin

40 (Fox, 2012). The force produced by myosin and actin in the sarcomere is termed the ensemble

41 force and can be calculated with the equation:

$$
F_{E N}=N_{t} \cdot f_{i} \cdot r, \quad(\mathrm{Eq} 1)
$$

43 where $N_{t}$ is the number of individual actomyosin interactions, $f_{i}$ is the force per actomyosin

44 interaction, and $r$ is the duty ratio of myosin (Sommese et al. 2013). The myosin duty ratio is the 45 proportion of the actomyosin ATPase cycle that myosin is bound to actin and can be calculated 46 using the equation:

$$
r=\left(\mathrm{k}_{\mathrm{cat}} \cdot \delta\right) / v_{o} \quad(\mathrm{Eq} 2)
$$

48 where $\mathrm{k}_{\mathrm{cat}}$ is the $k_{\text {cat }}$ of myosin's ATPase activity, $v_{o}$ is the velocity of the enzyme, and $\delta$ is the 49 step size of myosin (Howard 1997). 
The symptoms of HCM patients commonly include hyperdynamic systolic function

51 (Pandian et al. 2015), suggesting that sarcomere protein changes in HCM hearts result in

52 hypercontractility. However, current data does not sufficiently connect HCM-causing mutations

53 with hypercontractile sarcomeres (discussed in (Spudich 2014)). Nevertheless, changes in the

54 duty ratio of myosin will reflect changes in the contraction of HCM-affected hearts, with higher

55 duty ratios associated with hypercontractility.

HCM has been associated with 12 different $\alpha$-cardiac actin (ACTC) gene mutations.

57 Four ACTC variants found in HCM patients possess changes in or near the actomyosin-binding

58 site: H88Y, F904, R95C, and E99K (Figure 1A) (Olson et al. 2000, Morita et al. 2008, Kaski et

59 al. 2009, Behrmann et al. 2012). We previously examined the connection between HCM-related

60 human ACTC mutations and altered actomyosin interactions with 8 variants of ACTC, including

61 the E99K variant located in the actomyosin-binding site (Dahari and Dawson 2015). While the

62 E99K ACTC protein resulted in a higher duty ratio, we found that the other variants resulted in

63 duty ratios similar to wild-type ACTC protein. We hypothesized that the position of the amino

64 acid change on ACTC resulting from mutation impacts proteins binding at that site. For example,

65 the E99K substitution is located in the actomyosin binding site (Behrmann et al. 2012) and will

66 affect myosin function. R95 on ACTC may form a salt bridge with E576 and hydrogen bonds

67 with three other residues on myosin (Lorenz and Holmes 2010). With the loss of these

68 interactions, R95C ACTC might exhibit similar defects to those of E99K-ACTC. With H88Y

69 ACTC, the affected residue is on the same alpha helix in ACTC as R95 and E99. While more

70 distal from the actomyosin interface, an amino acid substitution may interfere with the packing

71 of residues along the helix, thereby indirectly inhibiting actomyosin interactions. Finally,

72 deletion mutants in actin are very rare, and the deletion of F90 in the helix containing R95 and 
73 E99 might alter the register and position of those amino acids, possibly disrupting interactions in

74 the actomyosin interface, resulting effects on actomyosin activity.

75 To test this hypothesis, we produced four HCM-related human ACTC variant proteins

76 with changes in or near the actomyosin binding site, including the E99K variant for comparison.

77 We calculated the duty ratios using myosin ATPase activity and in vitro motility assays, with the

78 expected result that all variants in the actomyosin binding site will exhibit increased myosin duty

79 ratios. Our work is the first biochemical analysis of the three subdomain-1 ACTC variant

80 proteins H88Y, F904, and R95C. Contrary to our expected results, we found that only E99K-

81 ACTC produced an increase in the duty ratio of myosin, suggesting that multiple factors play a

82 role in the development of HCM in the presence of other subdomain-1 ACTC variants.

\section{Materials and methods}

\section{Reagents and Basic Protocols}

86 All reagents were obtained from Thermo Fisher Scientific or Sigma-Aldrich, unless stated

87 otherwise. Recombinant purified ACTC protein concentrations were determined using Bio-Rad

88 protein assay dye and skeletal actin standards following the manufacturer's protocol (Hercules,

89 CA). $10 \%$ polyacrylamide resolving gels were used to conduct SDS-PAGE with a 5\% stacking

90 gel. Samples were mixed with 2x Laemmli buffer at a 1:2 ratio of buffer to sample (50 mM Tris,

$91 \mathrm{pH}$ 6.8, 2\% SDS, 10\% glycerol, $1.3 \mathrm{M} \beta \mathrm{ME}$ and 0.1\% bromophenol blue) (Laemmli 1970). Gels

92 were run for 45 minutes at $180 \mathrm{mV}$ with a $1 \mathrm{X}$ running buffer ( $25 \mathrm{mM}$ Tris, $250 \mathrm{mM}$ glycine, and

$930.1 \%$ SDS), and stained with Coomassie Stain and destained in 40\% methanol, 10\% acetic acid.

\section{Protein Purification}


Pure myosin and heavy meromyosin (HMM) was obtained from rabbit soleus muscle

97 (Margossian and Lowey 1982). The myosin obtained from the soleus muscle of rabbits is a combination of $>90 \%$ Type I (slow myosin; low ATPase activity) and $<10 \%$ Type II (fast myosin; high ATPase activity)(Billeter et al. 1980, Reiser et al. 1985). This is comparable to the myosin found in human cardiac ventricles which is a combination of $<10 \% \alpha$-myosin heavy 101 chain (fast) and $>90 \% \beta$-myosin heavy chain (slow)(Reiser et al. 2001, Narolska et al. 2005). $\alpha$-cardiac actin protein was prepared from the left ventricular muscle of bovine hearts

103 obtained from the Animal and Poultry Science Department (Guelph, ON) (Spudich and Watt 104 1971). Bovine $\alpha$-cardiac actin was used as a control as it shares $100 \%$ sequence identity to 105 human $\alpha$-cardiac actin protein. Bovine actin concentrations were determined by measuring 106 absorbance at $290 \mathrm{~nm}$ using an extinction coefficient of $0.62 \mathrm{~mL} \cdot \mathrm{mg}^{-1} \cdot \mathrm{cm}^{-1}$. Other protein 107 concentrations were determined with Bio-Rad protein dye reagent employing bovine actin as the 108 standard. Recombinant gelsolin and His-tagged G4-6 proteins were expressed and purified from

110 Escherichia coli following established protocols (Morrison and Dawson 2007, Ohki et al. 2009).

111 Recombinant human ACTC variant proteins were expressed in $S f 21$ cells with

112 recombinant baculoviruses (Mundia et al. 2012). To purify the recombinant ACTC proteins, we 113 adapted the affinity method of Ohki et al. (2009), binding actin proteins in cell lysates to His114 tagged G4-6 in the presence of calcium and eluting the ACTC proteins in an EGTA-containing 115 buffer. Cells from a $500 \mathrm{~mL}$ culture were first harvested by centrifugation and lysed in Buffer A 116 (10 mM Tris, $\mathrm{pH} 8.0,5 \mathrm{mM} \mathrm{CaCl}_{2}, 0.2 \mathrm{mM}$ PMSF, $0.01 \mathrm{mg} / \mathrm{mL}$ leupeptin, $1 \mathrm{mM} \mathrm{ATP,} 2 \mathrm{mM} \beta-$ 117 mercaptoethanol) by vortexing with glass beads (BioSpec Products) in a $50 \mathrm{~mL}$ conical tube for 1186 cycles of 1 minute vortexing followed by 4 minutes on ice. $10 \mathrm{mg}$ of His-G4-6 was then added 
119 to the lysate and mixed at $4^{\circ} \mathrm{C}$ overnight. After centrifugation at $25,000 \mathrm{rpm}$ in a TA 25.50 rotor

120 (Beckman) for 30 minutes, the supernatant was recirculated through a $5 \mathrm{~mL}$ Ni-NTA column

121 (Qiagen) in a closed loop for 1.5 hours. The column was then washed with $30 \mathrm{~mL}$ of Buffer A

122 containing $50 \mathrm{mM} \mathrm{KCl}$ followed by $40 \mathrm{~mL}$ of Buffer A containing $50 \mathrm{mM} \mathrm{KCl}$ and $20 \mathrm{mM}$

123 imidazole. A 1 mL HiTrap DEAE Sepharose FF column (GE Healthcare Life Science)

124 equilibrated in $10 \mathrm{mM}$ Tris, $\mathrm{pH}$ 8.0, 0.2 $\mathrm{mM} \mathrm{CaCl}_{2}, 1 \mathrm{mM}$ ATP, $0.125 \mathrm{mM}$ PMSF, $0.2 \mathrm{mM} \beta$ -

125 mercaptoethanol was attached to the bottom of the Ni-NTA column. $40 \mathrm{~mL}$ ACTC release buffer

126 (10 mM Tris, $\mathrm{pH}$ 8.0, $50 \mathrm{mM}$ KCl, $0.2 \mathrm{mM}$ EGTA, $0.2 \mathrm{mM}$ PMSF, $0.5 \mathrm{mM} \mathrm{MgCl} 2,0.2 \mathrm{mM}$

127 ATP, 2 mM $\beta$-mercaptoethanol) was applied to release the ACTC protein from the His-tagged

128 G4-6 bound on the Ni-NTA column. The released ACTC protein then bound to the DEAE

129 column. After disconnecting the DEAE column, the His-tagged G4-6 protein was eluted from the

130 Ni-NTA column in $15 \mathrm{~mL}$ G4-6 elution buffer (10 mM Tris, pH 8.0, $50 \mathrm{mM} \mathrm{KCl,} 0.2 \mathrm{M}$

131 imidazole, $\mathrm{pH}$ 8.0, $5 \mathrm{mM} \mathrm{CaCl}_{2}, 1 \mathrm{mM}$ PMSF, $1 \mathrm{mM}$ ATP, $2 \mathrm{mM} \beta$-mercaptoethanol). ACTC

132 was eluted from the DEAE column in ACTC elution buffer (10 mM Tris, $\mathrm{pH}$ 8.0, $0.2 \mathrm{mM} \mathrm{CaCl}_{2}$,

$1330.2 \mathrm{mM}$ ATP, $0.2 \mathrm{mM}$ PMSF, $0.2 \mathrm{mM} \beta$-mercaptoethanol, $0.3 \mathrm{M} \mathrm{KCl}$ ). ACTC-containing

134 fractions were dialyzed into G-buffer $(2 \mathrm{mM}$ Tris $\mathrm{pH} 8,0.2 \mathrm{mM} \mathrm{CaCl}, 0.2 \mathrm{mM} \mathrm{ATP}, 0.5 \mathrm{mM} \beta-$

135 mercaptoethanol, $0.002 \% \mathrm{NaN}_{3}$ ). Proteins were kept on ice and used within one week of

136 preparation. 10\% SDS-PAGE was used to verify the presence of pure ACTC and the progress of

137 purification. The average ACTC protein yields per $500 \mathrm{~mL}$ cell culture were as follows:

138 recombinant wild type (WTrec), $2.4 \pm 0.2 \mathrm{mg}(\mathrm{n}=22) ; \mathrm{H} 88 \mathrm{Y}$ ACTC, $4.3 \pm 0.4 \mathrm{mg}(\mathrm{n}=3) ; \mathrm{F} 90 \Delta$

139 ACTC, $2.4 \pm 0.1 \mathrm{mg}(\mathrm{n}=3) ; \mathrm{R} 95 \mathrm{C}$ ACTC, $3.1 \pm 0.3 \mathrm{mg}(\mathrm{n}=14) ;$ E99K ACTC, $2.3 \pm 0.3 \mathrm{mg}$ $140 \quad(n=6)$. 


\section{ATPase Assay}

The myosin ATPase activity assay used was a colorimetric assay based on the production

144 of a blue byproduct between released $\mathrm{P}_{\mathrm{i}}$ and molybdate, modified from Trybus (2000) for a 96-

145 well format. ACTC was polymerized in assay buffer (AB) $(75 \mathrm{mM} \mathrm{KCl}, 10 \mathrm{mM}$ imidazole, $\mathrm{pH}$

$1467,10 \mathrm{mM}$ DTT, $1 \mathrm{mM}$ EGTA, $1 \mathrm{mM} \mathrm{MgCl}_{2}$, and $1 \mathrm{mM} \mathrm{NaN}_{3}$ ), in the presence of a 1:1000

147 molar ratio of gelsolin to ACTC, and $2 \mathrm{mM} \mathrm{Mg-ATP.} \mathrm{Gelsolin} \mathrm{is} \mathrm{added} \mathrm{to} \mathrm{the} \mathrm{ATPase} \mathrm{assay} \mathrm{to}$

148 reduce viscosity of high F-actin concentrations, increasing the accuracy in the experiment

149 (Sommese et al. 2013). Samples of varying actin concentrations were produced through serial

150 dilutions in $\mathrm{AB}$ containing $2 \mathrm{mM} \mathrm{Mg-ATP}$ and kept on ice until they were added to the reaction.

151 The ATPase assay was performed using two 96 -well plates at $22^{\circ} \mathrm{C}$. The reaction plate

152 contained concentrations of $\mathrm{H}_{2} \mathrm{PO}_{4}$ ranging from $0.05 \mathrm{mM}$ to $2 \mathrm{mM}$ for a standard curve and

153 triplicate $100 \mu \mathrm{L}$ samples of each ACTC concentration. Rabbit soleus muscle myosin was

154 prepared in $1 \mathrm{X}$ AB without ATP to a final concentration of $0.125 \mathrm{mg} / \mathrm{mL} .100 \mu \mathrm{L}$ of myosin was

155 added to each actin sample well to begin the ATPase reaction (final concentration of 0.063

$156 \mathrm{mg} / \mathrm{mL}$ or $0.13 \mathrm{umol} / \mathrm{L}$ ). At 10 minute intervals for $40 \mathrm{~min}, 45 \mu \mathrm{L}$ of each reaction mixture was

157 removed and mixed with $45 \mu \mathrm{L}$ stop solution (60 mM EDTA pH 6.5, 6.6\% SDS) in wells of the

158 stop plate. Over the course of the assay, the ATPase rate was linear for all ATC variants (data

159 note shown). The standard samples were also mixed with $45 \mu \mathrm{L}$ stop solution.

After $40 \mathrm{~min}, 200 \mu \mathrm{L}$ of a molybdate colouring solution $(0.25 \mathrm{M} 2 \%$ ammonium

161 molybdate, $5 \mathrm{mg} / \mathrm{ml}$ ferrous sulfate) were added to the wells of the stop plate and the plate was

162 then read at $750 \mathrm{~nm}$ in a microplate reader. The standard curve was used to calculate the rate of

163 inorganic phosphate release in each assay. The average of the triplicate ATPase activities for

164 each concentration of actin was plotted against the concentration of actin in the assay and fit to 
165 the Michaelis-Menten curve using Prism software (GraphPad, La Jolla, CA). $k_{\text {cat }}$ was calculated

166 by determining the myosin concentration using a myosin heavy chain mass of $486 \mathrm{kDa}$.

167 Statistical analysis was accomplished using SPSS (IBM, Armonk, North Castle, NY).

\section{In Vitro Motility Assays}

The in vitro motility assay was adapted from Toyoshima (1987) and performed at $22^{\circ} \mathrm{C}$.

171 Active HMM was obtained by "deadheading" HMM with F-actin in the absence of ATP, co-

172 sedimentation of the acto-HMM complex, and release of active HMM in the presence of $2 \mathrm{mM}$

173 ATP. Flow cells were produced with nitrocellulose-coated coverslips and double-sided tape on

174 microscope slides where proteins were flowed in and incubated for 2 minutes for protein binding

175 and buffer exchanges. Deadheaded HMM diluted to $0.125 \mathrm{mg} / \mathrm{mL}$ in assay buffer was injected

176 first, followed by bovine serum albumin (BSA) diluted to $1 \mathrm{mg} / \mathrm{mL}$ in assay buffer to block the

177 nitrocellulose coverslip. The flow cell was then washed with assay buffer, and rhodamine

178 phalloidin-labelled ACTC protein variants (naked and/or regulated thin filaments) diluted to $\sim 0.1$

$179 \mu \mathrm{M}$ were bound to HMM in the nitrocellulose coverslip in the absence of ATP.

180 The flow cell was then washed with assay buffer prior to the addition of motility buffer

181 (MB; 25 mM KCl, 25 mM imidazole, pH 7.5, $10 \mathrm{mM}$ DTT, $4 \mathrm{mM} \mathrm{MgCl} 2,1 \mathrm{mM}$ EGTA, and 2

182 mM ATP) with an oxygen-scavenging system $(25 \mathrm{ug} / \mathrm{mL}$ glucose oxidase, $45 \mathrm{ug} / \mathrm{mL}$ catalase,

183 and $1 \%$ glucose) to prevent photobleaching. The flow cell was then inverted onto a Zeiss

184 Axiovert 200M (Zeiss, Jena, Germany) microscope and actin proteins were visualized at 100x

185 oil-immersed magnification using the Texas Red $(510 \mathrm{~nm})$ filter. The movement of filaments

186 was captured using a digital CCD ORCA-R2 C10600 camera (Hamamatsu, Middlesex, NJ) 
187 controlled by Volocity 6.3 software (Perkin Elmer, Waltham, MA) at a frame rate of 2.48

188 frames/second. Three movies at different locations on the nitrocellulose coverslip were captured.

189 The movement of ACTC filaments was tracked using a protocol adapted from Fraser and

190 Marston (1995). 14 to 20 single ACTC filaments moving in a linear pattern for at least 6 frames

191 were selected in each movie. Each filament's distance moved was tracked using Image J $1.47 \mathrm{v}$

192 and recorded on a customized Excel spreadsheet containing input of individual filament

193 coordinates, number of pixels moved per frame, frame rate displacement, and individual filament

194 velocities. The calibration of $89.5 \mathrm{~nm} /$ pixel was used to determine the total distance moved by

195 each filament.

197 Results

198 ACTC Purification

199 Previously, we used DNase-I affinity chromatography to purify recombinant human

200 ACTC proteins from insect cells infected with recombinant baculoviruses (Yates et al. 2009,

201 Mundia et al. 2012, Dahari and Dawson 2015). Here, we used recombinant His-tagged gelsolin

202 segments 4-6 (G4-6) to purify the human ACTC proteins in a calcium-dependent manner (Ohki

203 et al. 2009) using a combination of Ni-NTA columns for purification and DEAE columns for

204 actin concentration. Where DNase-I affinity chromatography requires slower-flowing resin and

205 harsh elution conditions containing 50\% formamide, the method employed here uses

206 commercially available fast-flow resin and mild buffer conditions, resulting in 2-4 mg of protein

207 from a $500 \mathrm{~mL}$ insect cell culture (Figure 1B).

208

209 Comparison of Tissue Purified and Recombinant ACTC 
To ensure that recombinant ACTC proteins purified with His-G4-6 protein possess the

211 same properties as tissue-purified cardiac actin, we compared actin-activated myosin ATPase

212 activity using bovine cardiac actin or recombinant WT ACTC (WTrec). The resulting Michealis-

213 Menten curves were similar (Figure 2 inset). Bovine cardiac actin produced a myosin ATPase

$214 \mathrm{~V}_{\max }$ of $14.9 \pm 1.0 \mu \mathrm{MP}_{\mathrm{i}} / \min$ and $\mathrm{K}_{\mathrm{M}}$ of $1.2 \pm 0.3 \mu \mathrm{M}$, while WTrec ACTC had a $\mathrm{V}_{\max }$ of $14.2 \pm$

$2151.0 \mu \mathrm{M} \mathrm{P} \mathrm{P}_{\mathrm{i}} / \mathrm{min}$ and $\mathrm{a} \mathrm{K}_{\mathrm{M}}$ of $1.0 \pm 0.4 \mu \mathrm{M}$.

216

217 The $\boldsymbol{k}_{\text {cat }}$ and Duty Ratio of ACTC Variants

218 Michaelis-Menten curves for the myosin ATPase activity of each ACTC variant were

219 generated (Figure 2A) and their $\mathrm{V}_{\max }$ and $\mathrm{K}_{\mathrm{M}}$ values determined by fitting the curves to the

220 Michaelis-Menten equation. The $\mathrm{V}_{\max }$ for each variant was used to determine the $k_{\text {cat }}$ of the

221 reaction given the amount of myosin employed (Table 1). No significant differences between

222 WTrec activities were seen compared to E99K and H88Y variants; there were significant

223 differences in comparing WTrec to F90 $\Delta$ and R95C.

224 In vitro motility assays determined the sliding velocity $v_{o}$ of each ACTC variant over a

225 bed of HMM (Figure 2B). There was a significant decrease in $v_{o}$ between WTrec $\left(v_{o}=1.99\right.$

$226 \mathrm{um} / \mathrm{sec})$ and E99K $\left(\mathrm{v}_{\mathrm{o}}=1.25 \mathrm{um} / \mathrm{sec}\right)$, consistent with previous studies (Debold et al. 2010,

227 Dahari and Dawson 2015). We also observed a significant increase between WTrec and F90 $\Delta$ $228 \quad\left(v_{o}=2.89 \mathrm{um} / \mathrm{sec}\right)$.

229 Assuming a fixed step size of $5 \mathrm{~nm}$ for myosin, we then calculated the duty ratio of each

230 ACTC variant, $r$, and the relative myosin duty ratio with respect to WTrec ACTC, $r_{r e l}($ Table 1).

231 E99K had an increased relative duty ratio of 1.36, consistent with our previous work (Dahari and 
232 Dawson 2015). Conversely, F904, and R95C had lower relative duty ratios of 0.43 and 0.71 ,

233 respectively, while H88Y had a duty ratio similar to WTrec.

234

\section{Discussion}

Previously, we characterized the intrinsic properties of several human ACTC variant

237 proteins and concluded that changes in subdomains- 1 and -4 of actin impact protein-protein 238 interactions (Mundia et al. 2012). We then determined the myosin duty ratio for a number of

239 ACTC variants located along the surface of actin, including E99K ACTC, concluding that

240 different molecular mechanisms exist for ACTC variants leading to HCM development (Dahari

241 and Dawson 2015). In the current work, we focused specifically on the actomyosin-binding site;

242 hypothesizing that ACTC variants in this region follow a similar dysfunctional pattern.

243 To test our hypothesis, we first purified recombinant human ACTC variant proteins

244 employing His-tagged G4-6 affinity chromatography (Ohki et al. 2009). We showed that tissue-

245 purified bovine ACTC protein and human WTrec protein did not exhibit any statistically

246 significant differences in myosin ATPase assays, indicating that the His-tagged G4-6 purification

247 method does not affect the activity of the resulting proteins.

248 We predicted that variants in the actomyosin binding site would follow the pattern of the

249 well-studied E99K ACTC variant, exhibiting an increase in myosin duty ratio; however, our data

250 from ATPase and in vitro motility assays showed variability between the actomyosin site of

251 other ACTC variants. In ATPase assays, E99K and H88Y ACTC variants exhibited activities

252 similar to WTrec, while R95C and F904 had lower ATPase activities. In the in vitro motility

253 assays, E99K ACTC had lower sliding velocities, while R95C and F90 $\Delta$ variants exhibited

254 higher velocities. The H88Y ACTC had a similar velocity to WTrec. Taken together, the 
255 calculated duty ratios based on a $5 \mathrm{~nm}$ myosin step size resulted in a duty ratio relative to WTrec

256 that was higher for E99K ACTC, consistent with our previous work (Dahari and Dawson 2015), 257 lower for R95C and F904 ACTC and similar for H88Y ACTC.

258 The hypothesis that a change in the same binding site will result in the same molecular 259 dysfunction does not account for the nature of that change. The E99K substitution results in a 260 charge-reversal at a site that is predicted to participate in a salt bridge with a counterpart amino 261 acid on myosin (Lorenz and Holmes 2010, Behrmann et al. 2012). Rather than form a salt bridge, 262 the charge reversal would result in charge repulsion at this site. H88Y is farther from the 263 predicted actomyosin atomic interactions and is a milder substitution. It is therefore unsurprising 264 that this ACTC variant exhibits properties similar to WTrec. The R95C change results in a loss 265 of charge in a potential salt bridge with myosin, similar to E99K, but it results in the opposite 266 molecular effects. Interestingly, the complete deletion of a codon encoding amino acid 90 in 267 ACTC might result in a change in the local structure and arrangement of amino acids in the 268 actomyosin binding site and results in a similar molecular dysfunction to R95C ACTC.

269 Despite being in the same molecular location on the actin molecule, the ACTC variants 270 studied here found in patients with HCM show different effects on actomyosin duty ratio. While 271 duty ratio is an important part of the overall contributors to cardiac contractility, ACTC variants 272 might also impact the step size $(\delta)$ or the intrinsic force $(f)$ of the actomyosin interaction

273 (Spudich 2014), resulting in changes in force production. To determine if these other parameters 274 are altered, single-molecule laser-trapping assays could be employed.

275 Our work benefits from being free of the complications of the regulatory machinery 276 found in cells; at the same time, those forms of regulation can compensate for changes in the 277 core force production between actin and myosin. Our approach starts with the core interactions; 
278 to gain a fuller picture, we also need to examine added layers of biological complexity involving

279 the products of other genes that are mutated in patients suffering HCM (Maron 2013), such as

280 examining regulated thin filaments in vitro or reconstituted thin filaments using sinusoidal

281 analysis (Bai et al. 2014, 2015). Integration of data from several levels of cardiac function

282 regulation will connect molecular defects to HCM development. These connections will allow us

283 to target specific changes in individual patients, tailoring treatments and leading to longer and

284 better lives.

285

286 Acknowledgements

287 We thank Matiyo Ojehomon, Navneet Sidhu, and Jordan Meyer for technical assistance.

288 This research was supported by a grant-in-aid from the Heart and Stroke Foundation of Canada.

289 J.D. is the recipient of a 2015 Heart and Stroke Foundation CP Has Heart Cardiovascular Award.

291 Author Contributions

292 J.D. provided lab space, supervision, supplies, preliminary research and wrote the

293 manuscript. H.L. provided $v_{o}$ from in vitro motility assays with ACTC variants used in the duty

294 ratio calculations. H.L. produced the baculoviruses containing ACTC mutants and infected the

295 insect cells with them. M.A. developed baculoviruses and completed preliminary datasets. M.H.

296 performed ATPase assays. 
297

298

299

300

301

302

303

304

305

306

307

308

309

310

311

312

313

314

315

316

317

318

319

\section{References}

Bai, F., Caster, H.M., Dawson, J.F., and Kawai, M. 2015. The immediate effect of HCM causing actin mutants E99K and A230V on actin-Tm-myosin interaction in thin-filament reconstituted myocardium. J. Mol. Cell. Cardiol. 79: 123-32. doi:10.1016/j.yjmcc.2014.10.014.

Bai, F., Caster, H.M., Rubenstein, P.A., Dawson, J.F., and Kawai, M. 2014. Using baculovirus/insect cell expressed recombinant actin to study the molecular pathogenesis of HCM caused by actin mutation A331P. J. Mol. Cell. Cardiol. 74: 64-75. Elsevier Ltd. doi:10.1016/j.yjmcc.2014.04.014.

Behrmann, E., Müller, M., Penczek, P.A., Mannherz, H.G., Manstein, D.J., and Raunser, S. 2012. Structure of the rigor actin-tropomyosin-myosin complex. Cell 150(2): 327-38. doi:10.1016/j.cel1.2012.05.037.

Billeter, R., Weber, H., Lutz, H., Howald, H., Eppenberger, H.M., and Jenny, E. 1980. Myosin types in human skeletal muscle fibers. Histochemistry 65(3): 249-259. doi:10.1007/BF00493174.

Dahari, M., and Dawson, J.F. 2015. Do cardiac actin mutations lead to altered actomyosin interactions? Biochem. Cell Biol. 93(4): 330-334. NRC Research Press. doi:10.1139/bcb2014-0156.

Debold, E.P., Saber, W., Cheema, Y., Bookwalter, C.S., Trybus, K.M., Warshaw, D.M., and VanBuren, P. 2010. Human actin mutations associated with hypertrophic and dilated cardiomyopathies demonstrate distinct thin filament regulatory properties in vitro. J. Mol. Cell. Cardiol. 48: 286-292. doi:10.1016/j.yjmcc.2009.09.014.

Fraser, I.D.C., and Marston, S.B. 1995. In vitro motility analysis of actin-tropomyosin regulation 
by troponin and calcium. The thin filament is switched as a single cooperative unit. doi:10.1074/jbc.270.14.7836.

322 Gajewski, K.K., and Saul, J.P. 2010. Sudden cardiac death in children and adolescents

323 (excluding Sudden Infant Death Syndrome). Ann. Pediatr. Cardiol. 3(2): 107-12. Medknow Publications and Media Pvt. Ltd. doi:10.4103/0974-2069.74035.

Howard, J. 1997. Molecular motors: structural adaptations to cellular functions. Nature 389(6651): 561-567. Nature Publishing Group. doi:10.1038/39247.

Kaski, J.P., Syrris, P., Esteban, M.T.T., Jenkins, S., Pantazis, A., Deanfield, J.E., McKenna, W.J., and Elliott, P.M. 2009. Prevalence of sarcomere protein gene mutations in preadolescent children with hypertrophic cardiomyopathy. Circ. Cardiovasc. Genet. 2(5): bacteriophage T4. Nature 227(5259): 680-685. Nature Publishing Group. doi: $10.1038 / 227680 \mathrm{a} 0$.

Lorenz, M., and Holmes, K.C. 2010. The actin-myosin interface. Proc. Natl. Acad. Sci. 107(28): 12529-12534. National Academy of Sciences. doi:10.1073/pnas.1003604107.

Maron, B.J., Ommen, S.R., Semsarian, C., Spirito, P., Olivotto, I., and Maron, M.S. 2014.

340 Hypertrophic Cardiomyopathy: Present and Future, With Translation Into Contemporary

341 Cardiovascular Medicine. J. Am. Coll. Cardiol. 64(1): 83-99.

342 doi:10.1016/j.jacc.2014.05.003. 
343 Maron, M.S. 2013. A Paradigm Shift in Our Understanding of the Development of the

344 Hypertrophic Cardiomyopathy Phenotype? Circulation 127(1). Available from

345 http://circ.ahajournals.org/content/127/1/10 [accessed 15 May 2017].

346 Morita, H., Rehm, H.L., Menesses, A., McDonough, B., Roberts, A.E., Kucherlapati, R.,

347 Towbin, J.A., Seidman, J.G., and Seidman, C.E. 2008. Shared Genetic Causes of Cardiac

348 Hypertrophy in Children and Adults. N. Engl. J. Med. 358(18): 1899-1908. Massachusetts

349 Medical Society . doi:10.1056/NEJMoa075463.

350 Morrison, S.S., and Dawson, J.F. 2007. A high-throughput assay shows that DNase-I binds actin

351 monomers and polymers with similar affinity. Anal. Biochem. 364(2): 159-64.

352 doi:10.1016/j.ab.2007.02.027.

353 Mundia, M.M., Demers, R.W., Chow, M.L., Perieteanu, A.A., and Dawson, J.F. 2012.

354 Subdomain location of mutations in cardiac actin correlate with type of functional change.

355 PLoS One 7(5): e36821. Public Library of Science. doi:10.1371/journal.pone.0036821.

356 Narolska, N.A., van Loon, R.B., Boontje, N.M., Zaremba, R., Penas, S.E., Russell, J.,

357 Spiegelenberg, S.R., Huybregts, M. a. J.M., Visser, F.C., de Jong, J.W., van der Velden, J.,

358 and Stienen, G.J.M. 2005. Myocardial contraction is 5-fold more economical in ventricular

359 than in atrial human tissue. Cardiovasc. Res. 65(1): 221-229.

360 doi:10.1016/j.cardiores.2004.09.029.

361 Ohki, T., Ohno, C., Oyama, K., Mikhailenko, S. V., and Ishiwata, S. 2009. Purification of

362 cytoplasmic actin by affinity chromatography using the C-terminal half of gelsolin.

363 Biochem. Biophys. Res. Commun. 383(1): 146-150. Elsevier Inc.

364 doi:10.1016/j.bbrc.2009.03.144.

365 Olson, T.M., Doan, T.P., Kishimoto, N.Y., Whitby, F.G., Ackerman, M.J., and Fananapazir, L. 
2000. Inherited and de novo Mutations in the Cardiac Actin Gene Cause Hypertrophic Cardiomyopathy. J. Mol. Cell. Cardiol. 32(9): 1687-1694. doi:10.1006/jmcc.2000.1204.

Pandian, N.G., Rowin, E.J., Gonzalez, A.M.G., and Maron, M.S. 2015. Echocardiographic profiles in hypertrophic cardiomyopathy: imaging beyond the septum and systolic anterior

Reiser, P.J., Moss, R.L., Giulian, G.G., and Greaser, M.L. 1985. Shortening velocity in single fibers from adult rabbit soleus muscles is correlated with myosin heavy chain composition. J. Biol. Chem. 260(16): 9077-9080.

Reiser, P.J., Portman, M.A., Ning, X.H., and Schomisch Moravec, C. 2001. Human cardiac myosin heavy chain isoforms in fetal and failing adult atria and ventricles. Am. J. Physiol. Heart Circ. Physiol. 280(4): H1814-1820. 8674(01)00242-2.

Sommese, R.F., Sung, J., Nag, S., Sutton, S., Deacon, J.C., Choe, E., Leinwand, L.A., Ruppel,

Spudich, J.A. 2014. Hypertrophic and Dilated Cardiomyopathy: Four Decades of Basic Research on Muscle Lead to Potential Therapeutic Approaches to These Devastating Genetic

Seidman, J.G., and Seidman, C. 2001. The Genetic Basis for Cardiomyopathy: from Mutation Identification to Mechanistic Paradigms. Cell 104(4): 557-567. doi:10.1016/S0092K., and Spudich, J.A. 2013. Molecular consequences of the R453C hypertrophic cardiomyopathy mutation on human $\beta$-cardiac myosin motor function. Proc. Natl. Acad. Sci. U. S. A. 110(31): 12607-12. doi:10.1073/pnas.1309493110.

Diseases. 106(6): 1236-49. The Biophysical Society. doi:10.1016/j.bpj.2014.02.011.

Spudich, J.A., and Watt, S. 1971. The Regulation of Rabbit Skeletal Muscle Contraction. J. Biol. Chem. 246(15): 4866-4871. 
389 Tarride, J.-E., Lim, M., DesMeules, M., Luo, W., Burke, N., O’Reilly, D., Bowen, J., and

390 Goeree, R. 2009. A review of the cost of cardiovascular disease. Can. J. Cardiol. 25(6):

391 e195-202. Pulsus Group. Available from http://www.ncbi.nlm.nih.gov/pubmed/19536390

$392 \quad$ [accessed 12 May 2017].

393 Trybus, K.M. 2000. Biochemical Studies of Myosin. Methods 22(4): 327-335.

394 doi:10.1006/meth.2000.1085.

395 WHO | Cardiovascular diseases (CVDs). 2016. World Health Organization. Available from 396 http://www.who.int/mediacentre/factsheets/fs317/en/ [accessed 12 May 2017].

397 Yates, S.P., Loncar, A., and Dawson, J.F. 2009. Actin polymerization is controlled by residue 398 size at position 204. Biochem. Cell Biol. 87(6): 853-65. doi:10.1139/o09-039.

399 Yoko Yano Toyoshima; Stephen J. Kron; Elizabeth M. Mcnally; Kenneth R. Niebling;

400 Chikashi Toyoshima; James A. Spudich. 1987. Myosin subfragment-1 is sufficient to move 401 actin filaments in vitro. Nature.

402 
Table 1: The relative duty ratio of myosin in the presence of ACTC variant proteins. Duty

406 ratios were determined using data from myosin ATPase assays and in vitro motility (IVM) data.

$407 V_{\max }, K_{M}$, and $v_{o}$ data are presented as the mean \pm standard error of the mean (SEM). Myosin

408 ATPase assays were performed in triplicate for two biological replicates. One biological

409 replicate was employed for E99K ACTC. For IVM assays, measurements were taken from 3

410 biological replicates (WTrec, H88Y, R95C) or 2 biological replicates (F904, E99K).

\begin{tabular}{|c|c|c|c|c|c|c|c|c|}
\hline \multicolumn{5}{|c|}{ Myosin ATPase assays } & \multicolumn{2}{|l|}{ IVM } & \multirow[b]{2}{*}{$\begin{array}{c}\text { Duty } \\
\text { ratio } \\
r\end{array}$} & \multirow[b]{2}{*}{$\begin{array}{c}\text { Relative } \\
\text { duty ratio } \\
r_{r e l}\end{array}$} \\
\hline Variant & $\begin{array}{c}V_{\max } \\
(\mu \mathrm{M} \\
\left.\mathrm{P}_{\mathrm{i}} / \min \right)\end{array}$ & $\begin{array}{c}k_{c a t} \\
\left(\min ^{-}\right. \\
1)\end{array}$ & $\begin{array}{c}K_{M} \\
(\mathrm{uM})\end{array}$ & $N$ & $\begin{array}{c}v_{o} \\
(\mu \mathrm{m} / \mathrm{sec})\end{array}$ & $\begin{array}{c}N \\
\text { (filaments } \\
\text { (assays)) }\end{array}$ & & \\
\hline WTrec & $\begin{array}{l}14.2 \\
\pm 1.0\end{array}$ & 55.13 & $1.9 \pm 0.4$ & 5 & $1.99 \pm 0.02$ & $180(9)$ & 0.14 & 1.00 \\
\hline H88Y & $\begin{array}{c}12.7 \pm \\
0.5\end{array}$ & 49.42 & $1.5 \pm 0.2$ & 6 & $1.84 \pm 0.03$ & $180(9)$ & 0.13 & 0.97 \\
\hline$R 95 C$ & $\begin{array}{c}11.2 \pm \\
1.3^{*}\end{array}$ & 43.46 & $3.5 \pm 0.9$ & 5 & $2.23 \pm 0.03$ & $218(11)$ & 0.10 & 0.71 \\
\hline F904 & $\begin{array}{l}8.8 \pm \\
0.4^{*}\end{array}$ & 34.40 & $1.1 \pm 0.2$ & 6 & $2.89 \pm 0.06^{\dagger}$ & $120(6)$ & 0.06 & 0.43 \\
\hline E99K & $\begin{array}{c}12.1 \pm \\
0.7\end{array}$ & 47.08 & $1.5 \pm 0.3$ & 3 & $1.25 \pm 0.02^{\dagger}$ & $89(5)$ & 0.19 & 1.36 \\
\hline
\end{tabular}

411

$412 *$ Statistically significant difference $(p<0.05)$ using a post hoc Dunnett's test.

$413 \dagger$ Statistically significant difference $(p<0.05)$ using a Kruskall-Wallis test.

414 


\section{Figure captions}

416 Figure 1: (A) A ribbon model of actin (PDB 1ATN) visualized using PyMol showing the

417 locations of HCM-related amino acid changes in the predicted myosin binding site. Bound ATP

418 is shown as sticks. The amino acid numbers listed are based on the sequence of actin following

419 posttranslational modification of the N-terminus removing the first two amino acids of the

420 nascent polypeptide. The PyMOL Molecular Graphics System, Version 1.3, Schrödinger, LLC.

421 (B) A progress of purification SDS-PAGE (10\%) of recombinant ACTC proteins from insect cell

422 culture using gelsolin segments 4-6 affinity chromatography. ACTC proteins in cell lysates are

423 bound to His-tagged G4-6 (lane 1) before being applied to a Ni-NTA column. The flow through

424 and resulting washes are shown (lanes 2-4). ACTC protein is released from the Ni-NTA resin in

425 a buffer containing EGTA and bound to a DEAE column attached in series. The bound G4-6

426 protein is eluted from the Ni-NTA column in high imidazole (lanes 5-6), while the ACTC protein

427 is eluted from the DEAE column with salt (lanes 7-9). Relative protein concentrations in elution

428 fractions were determined using Bio Rad protein dye in samples and the highest concentration

429 fractions were visualized on the gel. In both G4-6 and ACTC elutions, fraction 1 did not contain

430 a high concentration of protein and was not run on the gel. See Methods and materials for

431 details.

432

433 Figure 2: Biochemical analysis of ACTC variants. (A) Michaelis-Menten plots of recombinant

434 ACTC proteins. Best fit curve parameters are presented in Table 1. WTrec, H88Y, F90 , and

435 R95C ACTC plots represent data from 2 biological replicates with error bars representing the

436 SEM. E99K ACTC data is from 1 biological replicate with error bars representing SEM. inset,

437 Michaelis-Menten plots comparing WT ACTC protein from bovine cardiac tissue and WTrec 
438 proteins. Tissue-purified ACTC protein data is from 1 biological replicate. Post hoc Dunnet's

439 statistical testing was used to determine significant differences. (B) Box plots of in vitro motility

440 velocities of ACTC variants. Kruskal-Wallis statistical analysis showed there were no significant

441 differences between WTrec $(\mathrm{N}=9)$ to R95C $(p>0.05, \mathrm{~N}=11)$ and WTrec to H88Y $(p>0.05, \mathrm{~N}=$

442 9). There were significant differences betweenWTrec to F90 $\Delta\left({ }^{*} p<0.05, \mathrm{~N}=8\right)$, and WTrec to

$443 \quad \mathrm{E} 99 \mathrm{~K}(* p<0.05, \mathrm{~N}=5)$

444 

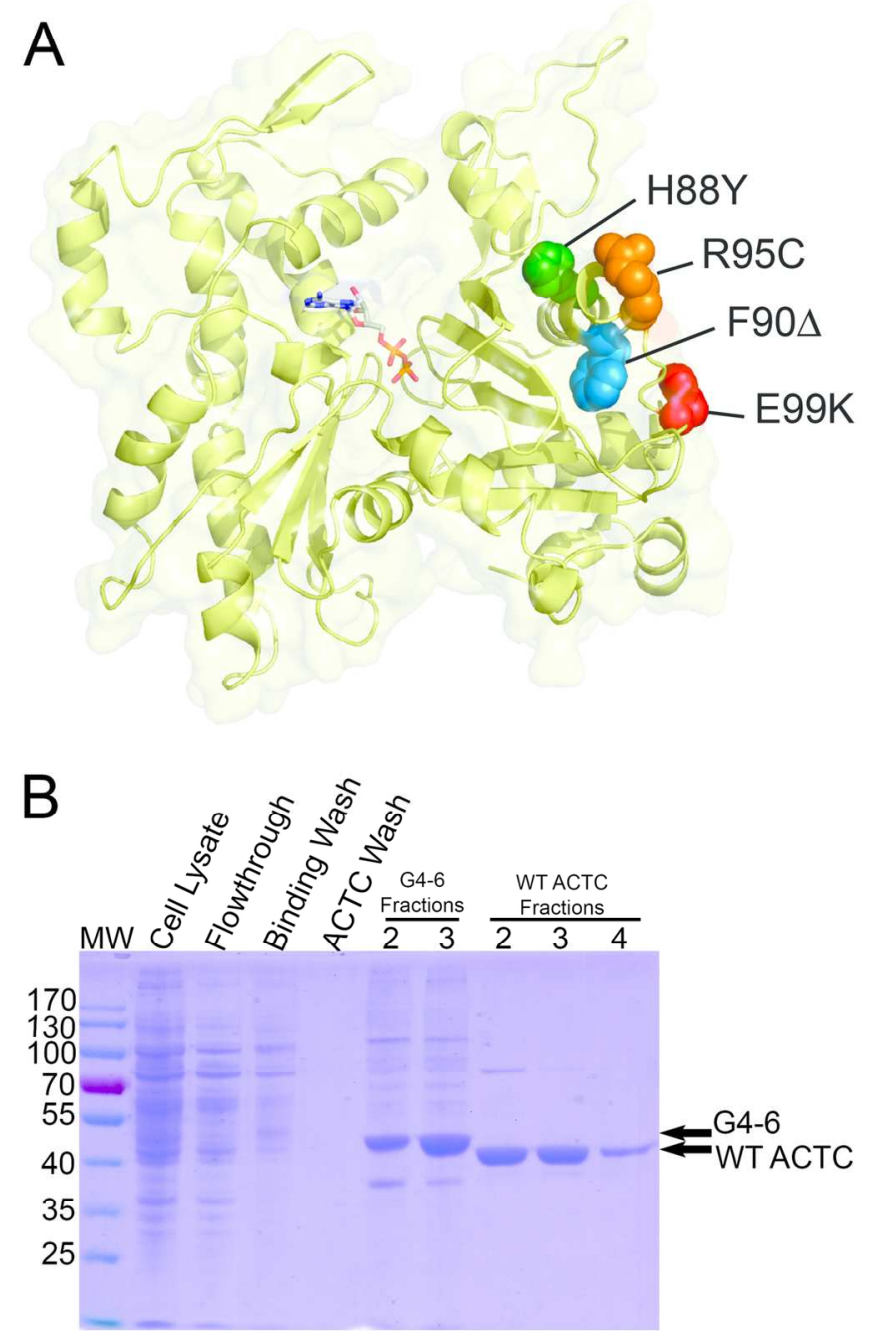

Figure 1: (A) A ribbon model of actin (PDB 1ATN) visualized using PyMol showing the locations of HCMrelated amino acid changes in the predicted myosin binding site. Bound ATP is shown as sticks. The amino acid numbers listed are based on the sequence of actin following posttranslational modification of the $\mathrm{N}$ terminus removing the first two amino acids of the nascent polypeptide. The PyMOL Molecular Graphics System, Version 1.3, Schrödinger, LLC. (B) A progress of purification SDS-PAGE (10\%) of recombinant ACTC proteins from insect cell culture using gelsolin segments 4-6 affinity chromatography. ACTC proteins in cell lysates are bound to His-tagged G4-6 (lane 1) before being applied to a Ni-NTA column. The flow through and resulting washes are shown (lanes 2-4). ACTC protein is released from the Ni-NTA resin in a buffer containing EGTA and bound to a DEAE column attached in series. The bound G4-6 protein is eluted from the $\mathrm{Ni}$-NTA column in high imidazole (lanes 5-6), while the ACTC protein is eluted from the DEAE column with salt (lanes 7-9). See Methods and materials for details.

$147 \times 160 \mathrm{~mm}(300 \times 300 \mathrm{DPI})$ 


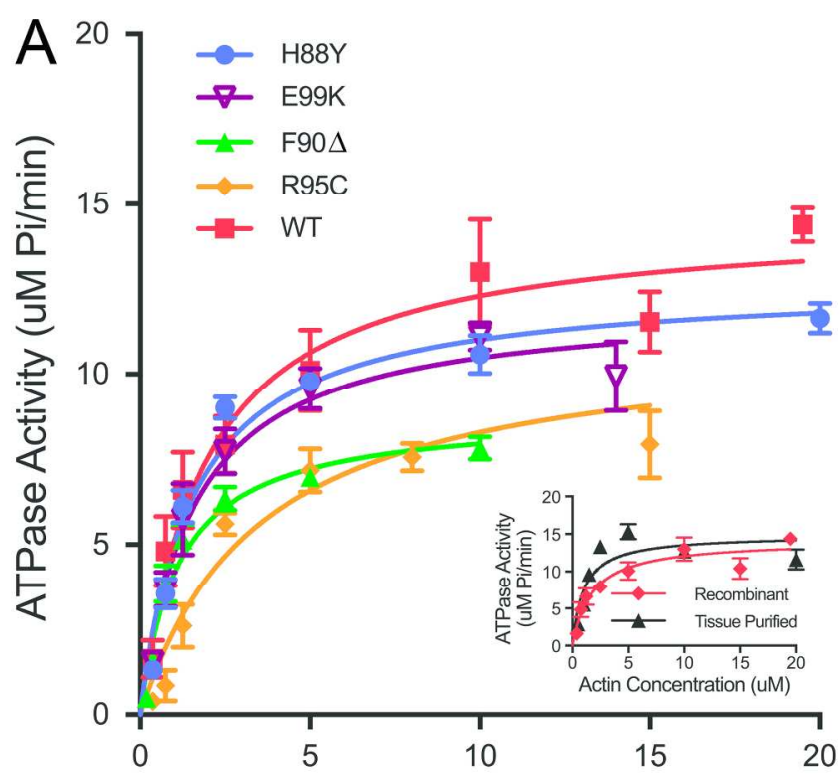

Actin Concentration (uM)

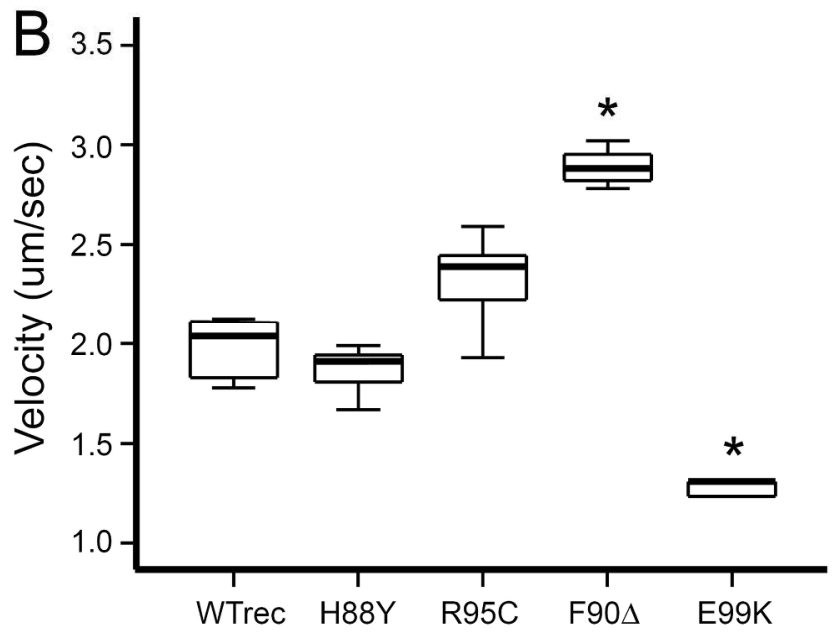

ACTC Variant

Figure 2: Biochemical analysis of ACTC variants. (A) Michaelis-Menten plots of recombinant ACTC proteins. Best fit curve parameters are presented in Table 1. WTrec, H88Y, F90 1 , and R95C ACTC plots represent data from 2 biological replicates with error bars representing the SEM. E99K ACTC data is from 1 biological replicate with error bars representing SEM. inset, Michaelis-Menten plots comparing WT ACTC protein from bovine cardiac tissue and WTrec proteins. Tissue-purified ACTC protein data is from 1 biological replicate. Post hoc Dunnet's statistical testing was used to determine significant differences. (B) Box plots of in vitro motility velocities of ACTC variants. Kruskal-Wallis statistical analysis showed there were no significant differences between WTrec $(\mathrm{N}=9)$ to R95C $(p>0.05, \mathrm{~N}=11)$ and WTrec to H88Y $(p>0.05, \mathrm{~N}=9)$. There were significant differences betweenWTrec to F90 $\Delta\left({ }^{*} p<0.05, \mathrm{~N}=8\right)$, and WTrec to E99K $(* p<0.05, \mathrm{~N}=5$.

$$
160 \times 276 \mathrm{~mm}(300 \times 300 \mathrm{DPI})
$$

